\title{
Thermally activated heat storage structures combined with a heat pump for the use in low energy buildings
}

\author{
Milan Ostrý, Tomáš Klubal, Roman Brzoň \\ Brno University of Technology, Faculty of Civil Engineering, Veveři 95, Brno 602 00, Czech Republic
}

\begin{abstract}
The building operating cost depends mainly on the quality of the building envelope and efficiency of systems for heating, cooling and domestic hot water. The big challenge for buildings with almost zero energy consumption is the use of renewable energy sources. The potential of renewable energy sources use for heating or cooling of buildings increases with the possibility to store heat or cold when they are available. Typical technologies utilize sensible heat storage technique, where the heat storage capacity depends mainly on the volume of medium and temperature differences. The capacity of storage medium, e.g. building structure, can be significantly increased by the use of proper kind of encapsulated Phase Change Materials (PCMs) for latent heat storage. Currently, the experiments carried out at Brno University of Technology are focused on latent heat storage technologies for proposed use in low- temperature heating or radiant cooling for residential and office buildings. The developed system uses air-water heat pump combined with sensible heat storage in storage tank and latent heat storage directly in building structures with integrated microencapsulated PCMs. Heating and cooling system consists of thermally activated floor, walls and ceiling structures. This system works with low temperature gradients in comparison with typical system based on the radiators situated bellow windows. The energy consumption and ability to fulfill the requirements on the quality of indoor environment of system described above are continuously observed and evaluated. Floor structure and plasterboards fixed on the ceiling and walls are thermally activated by capillary tubes. Water is used as a heat carrier medium. The floor slab and the plasterboards consist of $30 \mathrm{wt} \%$ of microencapsulated organic Phase Change Materials. The paper shows results from measurement in summer and winter period in special twin rooms and shows contribution of the system to reduction of operating cost of building.
\end{abstract}

Keywords: Radiant cooling; latent heat storage; building structure; thermal comfort; heat transfer

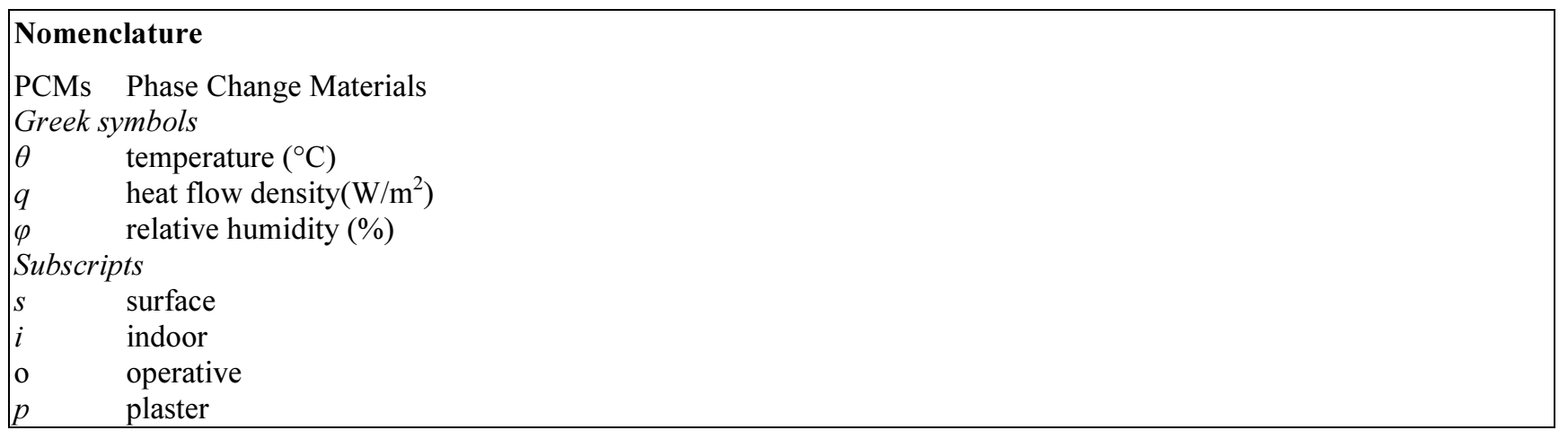

\section{Introduction}

The energy conservation in buildings is one of the main priorities in almost all developed countries. The energy needed for heating and cooling represents a significant part of energy consumption in residential buildings, office buildings and vast majority of other types of buildings. The energy consumption for heating and cooling also translates into a significant part of operation costs. For these reasons there is a permanent effort to apply energy saving measures reducing the energy consumption while maintaining or even improving thermal comfort of building occupants.

Corresponding author: Milan Ostrý. E-mail address: ostry.m@fce.vutbr.cz

http://dx.doi.org/10.3846/enviro.2014.277

(C) 2014 The Authors. Published by VGTU Press. This is an open-access article distributed under the terms of the Creative Commons Attribution License, which permits unrestricted use, distribution, and reproduction in any medium, provided the original author and source are credited. 
The thermally activated buildings' structures have been used in buildings for decades. The thermally activated building structures can be used for both heating and cooling and they have certain advantages for low-temperature heating and hightemperature cooling. These systems can employ low exergy heat sources which could not be used in other system (such as cooling and heating systems with fan-coils). The advantage of thermally activated building structures lies in the large surface area that allows for lower temperature difference in order to achieve the same heating or cooling output. Another advantage is the radiant heat transfer that makes it possible to achieve thermal comfort with lower indoor air temperatures during the heating season and higher indoor air temperatures in the cooling season. Of course, the thermally activated building structures have their performance limitations. The heating and cooling output per unit of area is limited and therefore the systems are able to deal with only a certain heating and cooling loads. It is possible to supplement thermally activated heating/cooling systems with other systems such as all-air air-conditioning systems but it makes economic sense in only certain cases. Utilization of thermally activated building structures for space heating in low energy buildings is usually not a problem. Their use for space cooling is also feasible when the internal heat gains are relatively small and the proper precautions are taken to reduce direct solar heat gains (shading devices). The increasing use of light weight-building structures with the high U-value building envelopes has brought about problems with overheating in summer and thus the need for cooling in the climates where cooling was not necessary in the past.

Koschenz and Lehmann [1] report the development of a thermally activated ceiling panel that could be used in lightweight and retrofitted buildings. The microencapsulated paraffin embedded in plaster was used in the panels. The behavior of the panels was investigated through both numerical simulations and the lab-scale experiments. The conclusion was that the panels containing $50 \mathrm{~mm}$ thick layer of gypsum-PCM mixture (with $25 \mathrm{wt}$ \% of microencapsulated PCM) were enough for the maintenance of comfortable room temperature in standard office buildings. Lehman et al. [2] presented a rather detailed study on the energy performance of a thermally activated building system (TABS) for an office building. They studied several variants of a TABS operating with three different control strategies. An evaporative cooling tower and a mechanical chiller with optional free cooling were considered as cold generation systems. A hydronic circuit topology (arrangement of distribution and return piping) played an important role in the energy efficiency of the TABS. A monovalent system with the cooling tower and separate zone return pipes showed the best energy performance in the study. One of the control strategies used in the study was the pulse width modulation (PWM) that is described in [3]. The pulse with modulation strategy makes use of intermittent operation of the circulation pump in order to save energy.

Pomianowski et al. [4] presented an experimental and numerical study into behavior of microencapsulated PCMs embedded in standard concrete. They found a significant discrepancy between heat capacity obtained theoretically and numerically. The authors come to the conclusion that theoretical assumptions about the performance of the concrete-PCM slabs are overestimated and the PCMs can actually decrease the cooling effect of a TABS. The authors state that further investigations are needed for optimal design and use of PCM-concrete TABS with regard to indoor temperature fluctuations.

The presented paper introduces thermally activated heat storage structures using an air-to-water heat pump as a heat source. The thermally activated heat storage structures and structure elements are the next step in the development of building integrated thermal storage for light-weight buildings that has been carried out at the Faculty of Civil Engineering in the last decade. Both experimental investigations and numerical simulations were performed in this area [5-7]. Cold storage in the building structures containing PCMs proved effective in reducing the cooling loads but it was found out that the rejection of heat (stored in the structures during the day) by intensive night-time ventilation was insufficient in hot summer days. The thermally activated structures (and structure elements) were proposed as a solution to this problem and tested in both lab-scale and full-scale experiments.

\section{Materials and Methods}

\subsection{Laboratory experiments}

The laboratory experiments focused on the comparison of heat transfer and heat storage capacity between different kinds of PCMs and different ways of their encapsulation. Because the sample size is relatively small, it was decided to use a small thermal incubator for a cyclic change of the boundary conditions (Fig. 1). Thermal incubator is equipped with the Peltier module and so the stability of the internal environment is linear and there are no fluctuations and any variability of the internal environment. The module itself operates on the principle of passing current circuit with two different conductors connected in series (bismuth and tellurium), where one of the contact surfaces is cooled and the other heated. The disadvantage is the higher energy consumption of this facility.

The gypsum plaster as a matrix was chosen for the first set of lab-scale experiments. The microcapsules filled with PCMs were mixed up with dry gypsum plaster. Microencapsulated Micronal®PCM was used in this mixture. The fraction of the microencapsulated PCM was $30 \mathrm{wt} . \%$. The matrix in the form of gypsum has a relatively high thermal conductivity and thus provides relatively good heat transfer between the ambient environment and the heat storage medium. Two samples with gypsum plaster were prepared for the testing. Sample "A" was made of the plain gypsum plaster without additives. Sample "B" was made of the gypsum plaster with addition of the heat storage medium - microencapsulated Micronal ${ }^{\circledR} P C M$ DS 5040X from BASF. Both samples were placed in the thermal incubator in the vertical position (Fig. 2). 


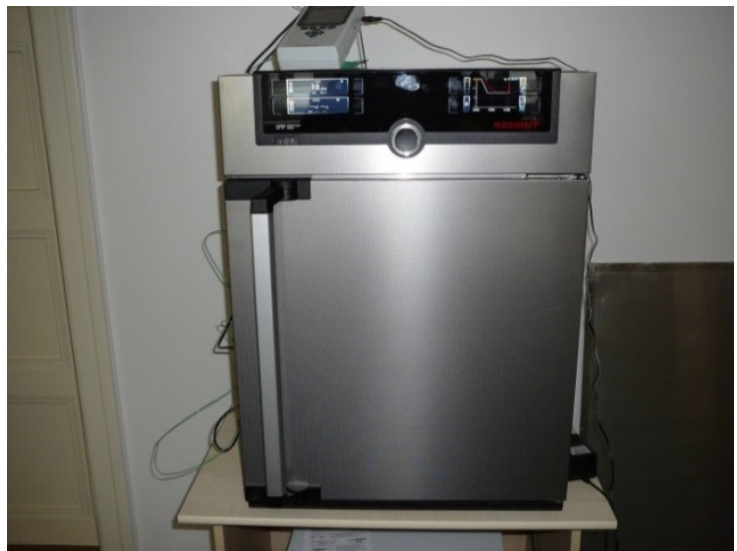

Fig. 1. View of thermal incubator

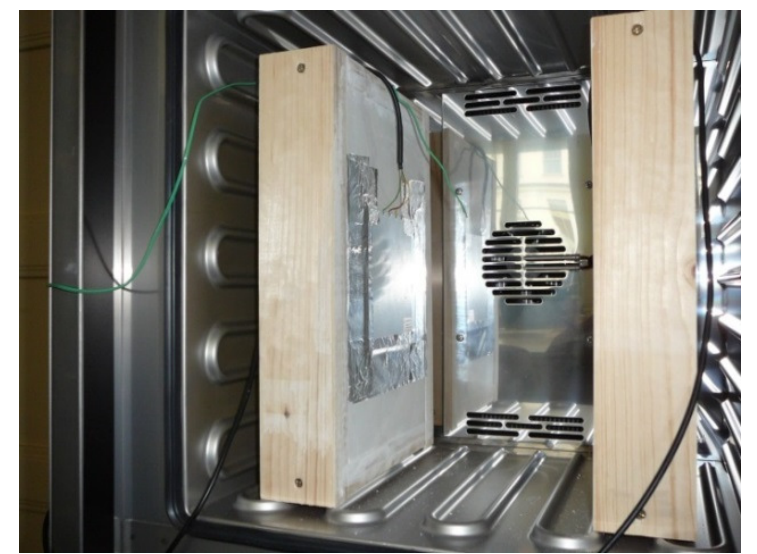

Fig. 2. Sample "A" and sample "B" situated in thermal incubator

There following values were monitored:

- indoor air temperature $-\theta_{i}$

- indoor air humidity $-\varphi_{i}$

- heat flux densities $-q$

- surface temperatures of the samples on the front and back side $-\theta_{s}$

\subsection{Full-scale experiments}

The full-scale experiments focused on the assessment of the influence of thermally activated heat storage panels on the indoor thermal comfort. Two identical test rooms are now available for comparative measurements at the Institute of Building Structures at Faculty of Civil Engineering. Both rooms have the same geometry, composition of the envelope and the orientation of skylights (Fig. 3). One of the test rooms was used as the referential room without heat storage structures and with a common air-conditioning unit; the other was the experimental room where thermally activated heat storage modules were installed and connected to a water circuit.

The heat storage modules were composed of a base layer and the layer of the modified plaster with the thickness of $15 \mathrm{~mm}$ (Fig. 4). The capillary tubes for thermal activations the modules were embedded in the plaster layer. The inlet and the outlet pipes were connected through the distributor and the collector to a heat pump that supplied the cooled water. The assembled panels were installed on the side walls, horizontal and sloping ceiling and on the floor. The air exchange in the experimental and the reference room was provided during the entire period by opening of the skylights to the ventilation positions. The volume of air inside each test room is approx. $30 \mathrm{~m}^{3}$.

There following values were observed in test rooms:

- operative temperature $-\theta_{o}$

- temperature inside plaster $-\theta_{p}$

- surface temperatures $-\theta_{s}$

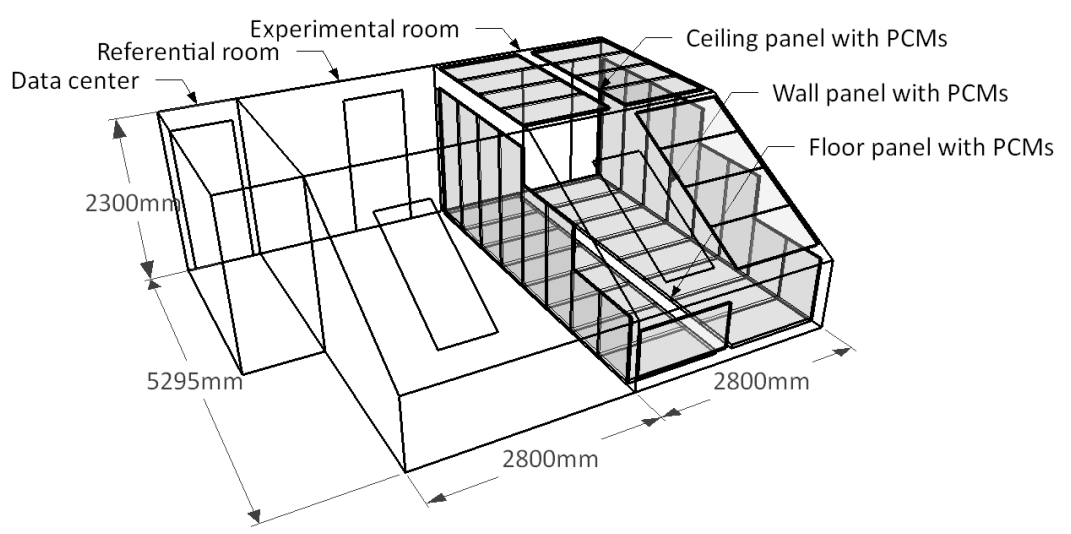

Fig. 3. The referential and experimental room for comparative measurements

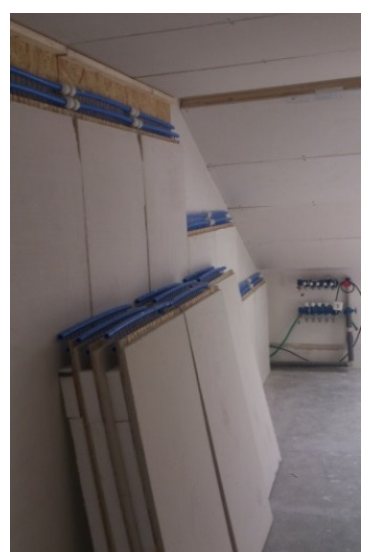

Fig. 4. Heat storage modules 


\section{Results and discussion}

\subsection{Laboratory experiments}

The lab-scale experiments were designed in a way to mimic the conditions in a real building with a small thermal storage capacity. A theoretical one-day cycle consisted of 4 stages (Fig. 5):

- Stage a - the constant temperature of $30^{\circ} \mathrm{C}$;

- Stage $\mathrm{b}$ - the linear decrease of temperature from $30^{\circ} \mathrm{C}$ to $10^{\circ} \mathrm{C}$;

- Stage c - the constant temperature of $10^{\circ} \mathrm{C}$;

- Stage $\mathrm{d}$ - the linear increase of temperature from $10{ }^{\circ} \mathrm{C}$ to $30^{\circ} \mathrm{C}$.

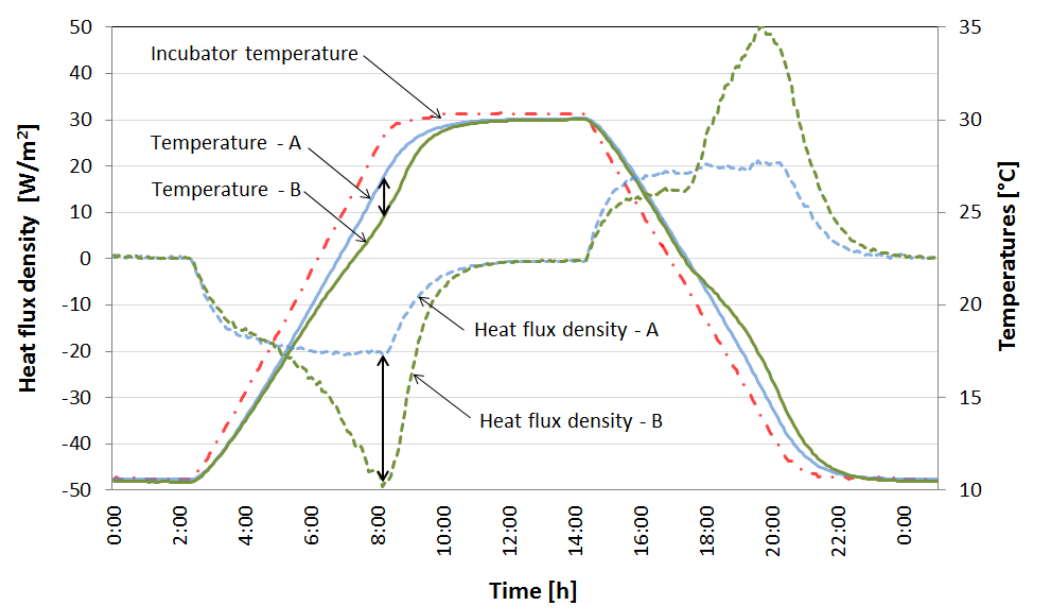

Fig. 5. Schematic of the differences between surface temperatures and heat flux densities for sample "A" and sample "B"

Fig. 5 shows the results for the one day cycle with heat storage and heat release periods. A significant difference between the heat storage capacities of the samples can be seen in the chart. The addition of heat storage capacity in the form of microencapsulated PCMs caused the heat flux density peaks during melting/freezing of PCMs and the differences of the surface temperatures. The process of heat storage and heat rejection was accompanied by the rise in the heat flux density.

\subsection{Full-scale experiments}

The comparative measurements of the effects of capillary cooling system coupled with a thermal storage layer containing PCMs were conducted in summer 2013. The effect on the indoor environment for different types of control of capillary cooling was observed. Various operation modes were tested during the experiments with the aim to minimize energy consumption and operating costs. Two modes of operation were studied:

- The panels were cooled down at night (cold storage) and they were cooled according to actual requirements on thermal comfort during the day. This operation is suitable for high cooling loads during the day;

- The panels were thermally activated at night (cold storage), with no cooling during the day. This mode of operation is suitable in summer without extreme values of maximum daily temperatures.

The first mode means activation of the panels (rejection of stored heat) at night and on-demand cooling during the day. The test rooms had the following operation profiles during the monitored period:

- Experimental room: the operation of the chiller was allowed by a timer in the following time intervals: 1:00 -1:30 a.m., 3:00-3:30 a.m. and 10:00 a.m. - 5:00 p.m. The chiller only switched on when the indoor temperature reached $25{ }^{\circ} \mathrm{C}$;

- Referential room: operation of air conditioning was enabled by a timer in the time interval between 10:00 a.m. and 5:00 p.m. Air conditioning only switched on when the indoor temperature reached $25^{\circ} \mathrm{C}$.

Fig. 6 shows the results for the time period of 2.8. - 3. 8. 2013. The operating time of the chiller for the experimental room and the air conditioning unit for the referential room was monitored. The air conditioning operated for 33 hours and 7 minutes and the chiller operated for 12 hours and 55 minutes in the total period of the test (188 hours). The chiller operated for only $40 \%$ of the operation time of air conditioning. The temperature in the experimental room oscillated between $23-26^{\circ} \mathrm{C}$. The temperature in the referential room increased when air conditioning was switched off and the maximum temperature in the referential room was more than $3{ }^{\circ} \mathrm{C}$ higher than the maximum temperature in the experimental room. The temperature of the plaster with and without PCMs in Fig. 6 shows the influence on latent heat storage. The temperature in the plaster with the PCMs rises significantly slower during melting of the PCMs (absorption of 
heat) than in case of the common plaster. During the cooling period the latent heat stored in the plaster with PCMs is released and thus the temperature does not drop as quickly and as low as in case of the common plaster.

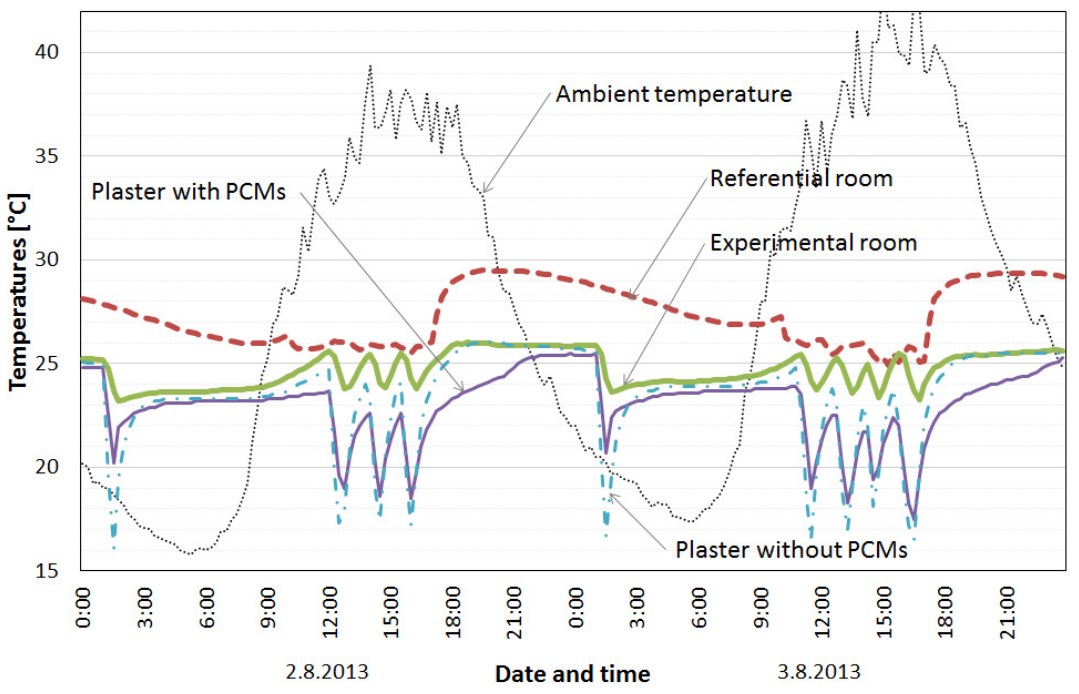

Fig. 6. Temperatures in the test rooms $(2.8 .-3.8 .2013)$

Another mode is the activation of PCMs at night. In the reported period the system worked in the following mode:

- Experimental room: at night from 3:00 to 3:30 a.m. the chiller (heat pump) was switched on to reject heat stored in the

PCMs (thermal activation of the panels). In this time the chiller started to work after indoor temperature reached $21{ }^{\circ} \mathrm{C}$.

The chiller was switched off during day;

- Referential room: air-conditioning was switched off all day.

The temperature in the experimental room was not actively controlled during the day. The panels containing PCMs and their thermal activation lasting only half an hour at night reduced the temperature peaks in the room by $2-3{ }^{\circ} \mathrm{C}$ (Fig. 7 ).

The Fig. 7 also shows the temperature inside the plaster. There is a gradual increase in temperature of the plaster with the PCMs, which is caused by latent heat storage upon reaching the melting temperature range. During the cooldown the heat stored in the PCMs is discharged and it results in a smaller temperature drop inside the plaster.

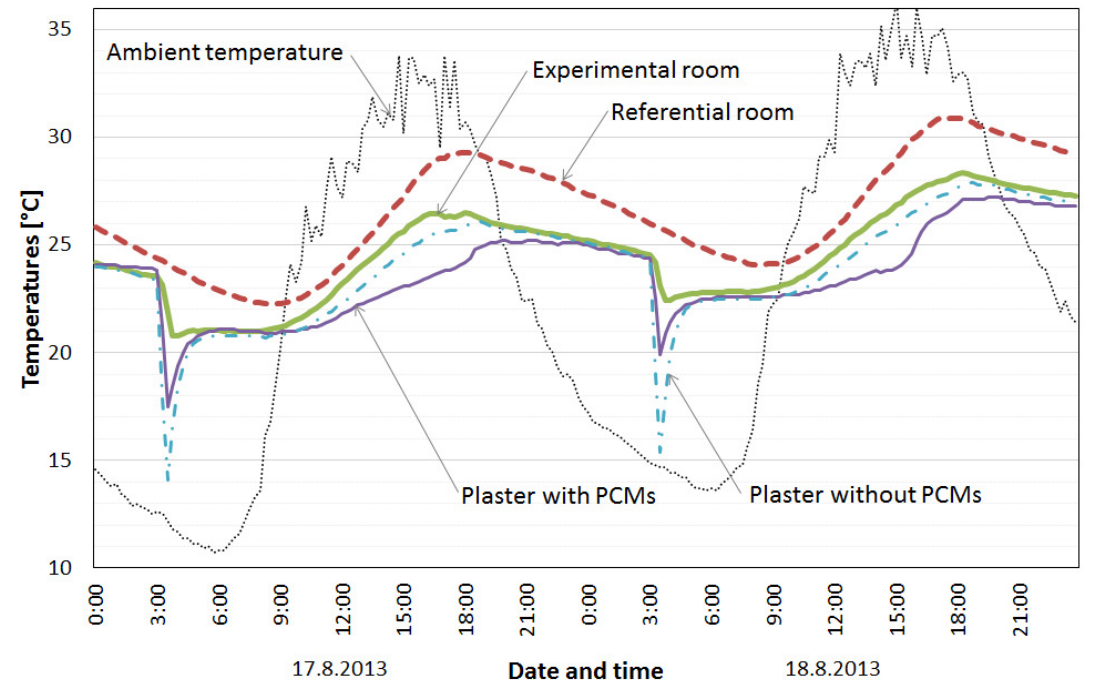

Fig. 7. Progressions of temperature in the testing rooms from 17. 8. - 18. 8.2013

\section{Conclusion}

Experimental investigation of the performance of thermally activated heat storage structures for space cooling was carried out. A sample of the heat storage module with PCMs was prepared and tested in a small thermal incubator (environmental chamber) together with the sample of light-weight structure. Consequently, the full-size thermally activated modules were prepared and installed in the experimental room. The thermally activated heat storage structures provided lower indoor temperature and better indoor temperature stability in the studied scenario than a fan-coil air-conditioning system. These results were proved by comparative measurement in test rooms. 


\section{Acknowledgements}

This work was supported by the Czech Grant Agency under project No. P104/12/1838 "Utilization of latent heat storage in phase change materials to reduce primary energy consumption in buildings" and by the project FAST-FSI-J-14-2232 "Effective capillary cooling with improved heat storage capacity."

\section{References}

[1] Koschenz, M.; Lehmann, B. 2004. Development of a thermally activated ceiling panel with PCM for application in lightweight and retrofitted buildings, Energy and Buildings 36: 567-578. http://dx.doi.org/10.1016/j.enbuild.2004.01.029

[2] Lehmann, B.; Dorer, V.; Gwerder, M.; Renggli, F.; Tödtli, J. 2011. Thermally activated building systems (TABS): Energy efficiency as a function of control strategy, hydronic circuit topology and (cold) generation system, Applied Energy 88: 180-191. http://dx.doi.org/10.1016/j.apenergy.2010.08.010

[3] Gwerder, M.; Tödtli, J.; Lehmann, B.; Dorer, V.; Güntensperger, W.; Renggli, F. 2009. Control of thermally activated building systems (TABS) in intermittent operation with pulse width modulation, Applied Energy 86: 1606-1616. http://dx.doi.org/10.1016/j.apenergy.2009.01.008

[4] Pomianowski, M.; Heiselberg, P.; Jensen, R. D. Dynamic heat storage and cooling capacity of a concrete deck with PCM and thermally activated building system, Energy and Buildings 53: 96-107.

[5] Charvát, P.; Klimeš, L. Ostrý, M., 2014. Numerical and experimental investigation of a PCM-based thermal storage unit for solar air systems, Energy and Buildings 68: 488-497. http://dx.doi.org/10.1016/j.enbuild.2013.10.011

[6] Charvat, P.; Mauder, T.; Ostry, M. 2012. Simulation of latent heat thermal storage integrated with the room structures, Materiali in tehnologije 46(3): 239-242.

[7] Klimeš, L.; Charvát, P.; Ostrý, M. 2012. Challenges in Computer Modeling of Phase Change Materials, Materiali in tehnologije 46(4): 335-338. 